\title{
Performance Analysis of Downlink NOMA System over Generalized Fading Channel
}

Link to publication record in Manchester Research Explorer

\section{Citation for published version (APA):}

Alqahtani, A., \& Alsusa, E. (2020). Performance Analysis of Downlink NOMA System over Generalized Fading Channel. 1-5. Paper presented at IEEE 91st Vehicular Technology Conference, Antwerp, Belgium.

https://ieeexplore.ieee.org/document/9128419

\section{Citing this paper}

Please note that where the full-text provided on Manchester Research Explorer is the Author Accepted Manuscript or Proof version this may differ from the final Published version. If citing, it is advised that you check and use the publisher's definitive version.

\section{General rights}

Copyright and moral rights for the publications made accessible in the Research Explorer are retained by the authors and/or other copyright owners and it is a condition of accessing publications that users recognise and abide by the legal requirements associated with these rights.

\section{Takedown policy}

If you believe that this document breaches copyright please refer to the University of Manchester's Takedown Procedures [http://man.ac.uk/04Y6Bo] or contact uml.scholarlycommunications@manchester.ac.uk providing relevant details, so we can investigate your claim.

\section{OPEN ACCESS}




\section{Performance Analysis of Downlink NOMA System over $\alpha-\eta-\mu$ Generalized Fading Channel}

\author{
Adel S. Alqahtani \\ School of Electrical and \\ Electronic Engineering \\ University of Manchester \\ Email: adel.alqahtani@postgrad.manchester.ac.uk
}

\author{
Dr. Emad Alsusa \\ School of Electrical and \\ Electronic Engineering \\ University of Manchester \\ Email: E.Alsusa@manchester.ac.uk
}

\begin{abstract}
In this paper, we evaluate the performance of NOMA system in downlink scenario over a well-known generalized fading channel called $\alpha-\eta-\mu$ in terms of the Average Bit Error Rate (ABER). The exact closed-forms of Average Bit Error Rate (ABER) for two NOMA users case are obtained in this work in order to study the significant of different fading parameters such as the non-linearity $(\alpha)$, the different power between the in-phase and quadrature components of the signal $(\eta)$, and the amount of multipath clusters $(\mu)$. Therefore, the Bit Error Rate (BER) of BPSK modulation technique is averaged by the probability of density function (PDF) of frequency flat fading channel modelled by $\alpha-\eta-\mu$ fading distribution. This work is being validated via a Monte Carlo Simulation to show the correction of the analytical derivations. However, this particular generalized fading channel can introduce other non-homogeneous attributes of the transmission medium such as Rayleigh, Weibull, Nakagami-m, $\alpha-\eta, \alpha-\mu$ fading channel models as special cases, which makes it more essential and worthy to be investigated.

Index Terms-Non-Orthogonal Multiple Access (NOMA), Average Bit Error Rate (ABER), $\alpha-\eta-\mu$ fading channels.
\end{abstract}

\section{INTRODUCTION}

The rapid revolution in wireless communication field has advocated a large amount of researchers to investigate more sophisticated issues in order to improve the system performance in different terms of concerns. Therefor, it becomes one of the most controversial research area that essentially includes variety aspects of research challenges such as the multiple access techniques among users equipments and base stations. Consequently, Non-Orthogonal Multiple Access (NOMA) technique is considered as a well known candidate technology to the future mobile generation due to its promising features. However, in the cellular system, some of the major challenges in the propagation medium that unfavorably degrade the radio wave signal's quality known as multipath fading and shadowing effect have been widely devoted in the previous generations. Moreover, some statistical models such as Rayleigh, Rice, Nakagami-m, and other fading models can straightforwardly characterize the multipath fading in line of site (LOS) and nonline of site (NLOS) scenarios because of its applicability and traceability. On the contrary, obtaining an exact closed form for different performance metrics of the orthogonal wireless transmission system such as the outage probability, the average channel capacity, the average bit error rate, the amount of fading and other performance measurements are being studied widely in the literature, and they are considered as a mathematical challenges specifically when it comes to a more generalized fading model such as $\alpha-\eta-\mu$ model [1], [2]. Novelty studies on evaluating wireless system performance have covered numerous types of fading distributions. For instance, authors in [3] have analyzed and evaluated the outage probability of $\eta-\mu$ fading channel in a closed form expression while in [4] the average symbol error rate (SER) of the same fading model in terms of Meijer $\mathrm{G}$ function have been evaluated. Moreover, Yacoub has investigated different types of generalized fading distributions called $\alpha-\mu, \eta-\mu, \lambda-\mu$, and $\kappa-\mu$ in [5], [6]. In addition, a new remarkable generalized fading model known as $\alpha-\eta-\mu$ fading distribution is being provided by Yacoub in [7], in which it includes most of the aforementioned fading models as special cases.

However, to the best of our knowledge, there is no performance evaluation of NOMA system over $\alpha-\eta-\mu$ fading distribution has been done in the literature before. Therefor, in this paper, we intend to devote the performance analysis of two NOMA users case over $\alpha-\eta-\mu$ fading channel which renders the other fading models such as the Rayleigh, the Weibull, the Nakagami-m, the $\alpha-\eta$, the $\alpha-\mu$ fading distributions as special cases. Exact closed forms of the average BER of both users will be evaluated and validated by the Matlab simulation as well. The remaining of this paper is organized as follow: in section II, we introduce the system model of DL-NOMA system. In section III, the $\alpha-\eta-\mu$ generalized fading distribution is defined and explained along with the derivation of the average Bit Error Rate (ABER) of two NOMA users. Moreover, the numerical and simulation results are presented in section IV. Ultimately, the conclusion of this work will be given in section V.

\section{SySTEM MODEL}

Consider a downlink NOMA transmission system applied for two active users served by a single base station (BS) which radiates broadcast signals simultaneously for both users on the same frequency and time slots by performing the superposition coding scheme. Both users transmitted signals will be allocated to some particular amount of power, and then aggregated to be propagated as a single stream in downlink scenario. The received signal at each user's terminal can be represented as:

$$
y_{i}=h_{i} x_{s c}+n_{i},
$$


where $h_{i}$ indicates the channel gain between the users and the BS, and $h_{i}=\left|\bar{g}_{i}\right|^{2} / \sqrt{1+d_{i}^{l}}$, where $g_{i}$ is the fading channel gain, and $l$ refers to the path-loss factor while $d_{i}$ indicates the distance between the user and the BS. Moreover, according to the NOMA concept, it is assumed that cell edge user (U1) experiences very poor channel gain than cell center user (U2) i.e, $\left|h_{1}\right|<\left|h_{2}\right|$. Consequently, the allocation power of both users will be distributed as $\left(\varepsilon_{1}>\varepsilon_{2}\right)$, where $\varepsilon_{1}=\alpha P_{t}$, and $\varepsilon_{2}=(1-\alpha) P_{t}$, and $P_{t}$ and $\alpha$ are the total transmitted power, and the allocated power coefficient, respectively. In addition, $n_{i}$ represents the additive white Gaussian noise (AWGN), and $x_{s c}$ is the superimposed signal which is $x_{s c}=\sum_{i=1}^{k} \sqrt{\varepsilon_{i}} x_{i}$, where $k$ refers to the number of users and $x$ indicates the modulated symbol which will be allocated to some a mount of energy $\varepsilon_{i}$. It is also assumed that modulation technique for both user is based on the Binary Phase Shift Keying (BPSK) modulation type.

\section{Performance Analysis of The $\alpha-\eta-\mu$ GENERALIZED FADING CHANNEL}

The $\alpha-\eta-\mu$ distribution is considered when there is no line of sight (NLOS), and introduced by three major natures of fading components represented in the non-linearity $(\alpha)$, the different power between the in-phase and quadrature components of the signal $(\eta)$, and the amount of multipath clusters $(\mu)$. This particular type of fading distribution might be examined in two different format using its PDF of the instantaneous signal to noise ratio $(\gamma)$, which is given as below:

$$
\begin{aligned}
& P_{\gamma}(\gamma)=\frac{\sqrt{\pi} \alpha \mu^{\mu+\frac{1}{2}} h^{\mu} \gamma^{\frac{\alpha}{2}\left(\mu+\frac{1}{2}\right)-1}}{\Gamma(\mu) H^{\mu-\frac{1}{2}} \tilde{\gamma}^{\frac{\alpha}{2}\left(\mu+\frac{1}{2}\right)}} \\
& \quad \times \exp \left(-\frac{2 \mu h \gamma^{\frac{\alpha}{2}}}{\tilde{\gamma}^{\frac{\alpha}{2}}}\right) \times I_{\mu-\frac{1}{2}}\left(\frac{2 \mu H \gamma^{\frac{\alpha}{2}}}{\tilde{\gamma}^{\frac{\alpha}{2}}}\right),
\end{aligned}
$$

where $\tilde{\gamma}$ is the average $\operatorname{SNR}(\gamma), \Gamma($.$) is the gamma function,$ $I_{v}($.$) indicates to the modified Bessel function of the first kind$ where $\mathrm{v}$ is the first arbitrary order, the nonlinear propagation power is captured by $\alpha>0$, and the number of multipath clusters is indicated by $\mu>0$. However, as it has been mentioned above that this type of fading model could be defined in two different formats, Format I: $\left(h=\frac{(1+\eta)^{2}}{4 \eta}\right)$ and $\left(H=\frac{1-\eta^{2}}{4 \eta}\right)$, where $(0<\eta<\infty)$ indicates the power of the scattered wave between the in-phase and quadrature components. Format II $:\left(h=\frac{1}{1-\eta^{2}}\right)$ and $\left(H=\frac{\eta}{1-\eta^{2}}\right)$, where $(-1<\eta<1)$ represents the correlation factor between the in-phase and quadrature components.

\section{A. Average BER of U1 ( Cell Edge User) over $\alpha-\eta-\mu$ Fading Channel:}

In order to find the Average BER, we initially compute the conditional BER of U1 based on the decision boundaries of the maximum likelihood detection on its superimposed constellation points that implemented over BPSK modulation technique, and then perform the averaging process mentioned in [11, eq. 1.8] such as:

$$
\bar{P}_{U_{i}}(e)=\int_{0}^{\infty} P_{U_{i}}(e) \cdot P_{\gamma}(\gamma) d \gamma .
$$

Therefore, the conditional BER based BPSK modulation of the cell edge user (U1) can be expressed in the following form:

$$
P_{U 1}(e)=\frac{1}{2} \times\left[Q\left(\sqrt{\gamma_{1}}\right)+Q\left(\sqrt{\gamma_{2}}\right)\right] .
$$

Notice that some details on eq.(4) are omitted to conserve space. Now, we plug equation (2) and (4) in (3), which yields:

$$
\begin{aligned}
\bar{P}_{U 1}(e) & =\frac{1}{2} \times \sum_{i=1}^{2} \frac{\sqrt{\pi} A_{c} \alpha \mu^{\mu+\frac{1}{2}} h^{\mu}}{\Gamma(\mu) H^{\mu-\frac{1}{2}} \tilde{\gamma}_{i}^{\frac{\alpha}{2}\left(\mu+\frac{1}{2}\right)}} \int_{0}^{\infty} \gamma_{i}^{\frac{\alpha}{2}\left(\mu+\frac{1}{2}\right)-1} \\
& \times \exp \left(-\frac{2 \mu h \gamma_{i}^{\frac{\alpha}{2}}}{\tilde{\gamma}_{i}^{\frac{\alpha}{2}}}\right) \times I_{\mu-\frac{1}{2}}\left(\frac{2 \mu H \gamma_{i}^{\frac{\alpha}{2}}}{\tilde{\gamma}_{i}{ }^{\frac{\alpha}{2}}}\right) \operatorname{erfc}\left(\sqrt{\gamma_{i}}\right) d \gamma_{i},
\end{aligned}
$$

where $i$ indicates the user's number which in this case belongs to $\{1,2\}$, and $\gamma_{1}=\sqrt{E_{s_{1}} / 2 \sigma_{1}^{2}}+\sqrt{E_{s_{2}} / 2 \sigma_{1}^{2}}$, and $\gamma_{2}=\sqrt{E_{s_{1}} / 2 \sigma_{1}^{2}}-\sqrt{E_{s_{2}} / 2 \sigma_{1}^{2}}$ , where $E_{s_{i}}$ and $\sigma_{i}$ indicate the user symbol energy and noise variance, respectively. Now, we further simplify eq. (5) by implementing the Laplace transform that has been found in [2] in order to efficiently solve the inner integral in the above equation. Thus, eq.(5) becomes like :

$$
\begin{aligned}
\bar{P}_{U 1}(e) & =\frac{1}{2} \times \sum_{i=1}^{2} \frac{\sqrt{\pi} A_{c} \alpha \mu^{\mu+\frac{1}{2}} h^{\mu}}{\Gamma(\mu) H^{\mu-\frac{1}{2}} \tilde{\gamma}_{i}^{\frac{\alpha}{2}\left(\mu+\frac{1}{2}\right)}} \\
& \times \mathscr{L}\left\{\gamma_{i}^{\left(\mu+\frac{1}{2}\right)-1} I_{\mu-\frac{1}{2}}\left(\frac{2 \mu H \gamma_{i}}{\tilde{\gamma}_{i}^{\frac{\alpha}{2}}}\right)\right. \\
& \left.\times \operatorname{erfc}\left(\sqrt{B_{c}} \gamma_{i}^{\frac{1}{\alpha}}\right),\{\gamma, p\}\right\} .
\end{aligned}
$$

$\mathscr{L}$ (.) is the Laplace transform component where $p=2 \frac{\mu h}{\tilde{\gamma}_{i}^{\frac{\alpha}{2}}}, p \in$ $\{0, \infty\}$. Now, by using the aid of the Laplace transform mentioned in table III in [2] and the Eulerian Integrals for the $\mathrm{H}$ function in [12, eq. 2.57], the appropriate final formula will be found and written in the following format:

$$
\begin{aligned}
\bar{P}_{U 1}(e) & =\frac{1}{2} \times \sum_{i=1}^{2} \frac{A_{c} \mathscr{A}_{i}}{\sqrt{\pi} B_{c}^{\frac{\alpha}{2}\left(\mu+\frac{1}{2}\right)}}\left(\frac{1}{2 \pi l}\right)^{2} \\
& \int_{L 1} \int_{L 2} \Gamma(\xi 1) *\left(\frac{2 \mu(h-H)}{\tilde{\gamma}_{i}^{\frac{\alpha}{2}}}\right)^{-\xi 1} \times \\
& \frac{\Gamma\left(\mu-\frac{1}{2}+\xi 2\right) \Gamma\left(\frac{1}{2}-\xi 2\right)}{\Gamma\left(\frac{1}{2}+\mu-\xi 2\right)}\left(\frac{4 \mu H}{\tilde{\gamma}_{i}^{\frac{\alpha}{2}}}\right)^{-\xi 2} \\
& \times \underbrace{\int_{0}^{\infty} \gamma^{\frac{\alpha}{2}\left(\mu+\frac{1}{2}-\xi 1-\xi 2\right)-1} \operatorname{erfc}\left(\sqrt{B_{c} \gamma_{i}}\right) d \gamma_{i} d \xi 1 d \xi 2} .
\end{aligned}
$$




$$
\begin{aligned}
\bar{P}_{U 1}(e) & =\frac{1}{2} \times \\
& \sum_{i=1}^{2} \frac{A_{c} \alpha \mu^{\mu+\frac{1}{2}} h^{\mu}}{\sqrt{\pi} H^{\mu-\frac{1}{2}} \Gamma(\mu)\left(B_{c} \tilde{\gamma}_{i}\right)^{\frac{\alpha}{2}\left(\mu+\frac{1}{2}\right)}} H_{2,1: 0,1 ; 1,2}^{0,2: 1,0 ; 1,1}\left[\begin{array}{cc}
\frac{2 \mu(h-H)}{\left(B_{c} \gamma_{i}\right) \frac{\alpha}{2}} & \mid\left(\frac{1}{2}-\frac{\alpha}{2}\left(\mu+\frac{1}{2}\right) ; \frac{\alpha}{2}, \frac{\alpha}{2}\right),\left(1-\frac{\alpha}{2}\left(\mu+\frac{1}{2}\right) ; \frac{\alpha}{2}, \frac{\alpha}{2}\right):-;\left(\frac{1}{2}, 1\right) \\
\frac{4 \mu H}{\left(B_{c} \gamma_{i}\right)^{\frac{\alpha}{2}}} \mid & \left(-\frac{\alpha}{2}\left(\mu+\frac{1}{2}\right) ; \frac{\alpha}{2}, \frac{\alpha}{2}\right):(0,1) ;\left(\mu-\frac{1}{2}, 1\right),\left(\frac{1}{2}-\mu, 1\right)
\end{array}\right]
\end{aligned}
$$

$$
\begin{aligned}
& \bar{P}_{U 2}(e)=\frac{1}{2} \times \\
& \sum_{i i=1}^{5} \frac{A_{c} \alpha \mu^{\mu+\frac{1}{2}} h^{\mu}}{\sqrt{\pi} H^{\mu-\frac{1}{2}} \Gamma(\mu)\left(B_{c} \tilde{\gamma}_{i i}\right)^{\frac{\alpha}{2}\left(\mu+\frac{1}{2}\right)}} H_{2,1: 0,1 ; 1,2}^{0,2: 1,0 ; 1,1}\left[\begin{array}{cc}
\frac{2 \mu(h-H)}{\tilde{\gamma}_{i i}^{\frac{\alpha}{2}}} & \left(\frac{1}{2}-\frac{\alpha}{2}\left(\mu+\frac{1}{2}\right) ; \frac{\alpha}{2}, \frac{\alpha}{2}\right),\left(1-\frac{\alpha}{2}\left(\mu+\frac{1}{2}\right) ; \frac{\alpha}{2}, \frac{\alpha}{2}\right):-;\left(\frac{1}{2}, 1\right) \\
\frac{4 \mu H}{\tilde{\gamma}_{i i}^{\frac{\alpha}{2}}} & \left(-\frac{\alpha}{2}\left(\mu+\frac{1}{2}\right) ; \frac{\alpha}{2}, \frac{\alpha}{2}\right):(0,1) ;\left(\mu-\frac{1}{2}, 1\right),\left(\frac{1}{2}-\mu, 1\right)
\end{array}\right]
\end{aligned}
$$

$$
\begin{aligned}
P_{U 2}(e) & =\frac{1}{2} \times\left[-Q\left(\sqrt{\gamma_{A}}\right) \cdot f_{\gamma_{A}}\left(\gamma_{A}\right) d \gamma_{A}+Q\left(\sqrt{\gamma_{B}}\right) \cdot f_{\gamma_{B}}\left(\gamma_{B}\right) d \gamma_{B}+Q\left(\sqrt{\gamma_{C}}\right) \cdot f_{\gamma_{C}}\left(\gamma_{C}\right) d \gamma_{C}\right. \\
& \left.-Q\left(\sqrt{\gamma_{D}}\right) \cdot f_{\gamma_{D}}\left(\gamma_{D}\right) d \gamma_{D}+Q\left(\sqrt{\gamma_{E}}\right) \cdot f_{\gamma_{E}}\left(\gamma_{E}\right) d \gamma_{E}+Q\left(\sqrt{\gamma_{B}}\right) \cdot f_{\gamma_{B}}\left(\gamma_{B}\right) d \gamma_{B}\right]
\end{aligned}
$$

The underlined integral in equation (7) can be solved as in [2, eq. 23], which eventually will produce the final formula of ABER of the cell edge user (U1) as the following:

$$
\begin{aligned}
\bar{P}_{U 1}(e) & =\frac{1}{2} \times \sum_{i=1}^{2} \frac{A_{c} \mathscr{A}_{i}}{\sqrt{\pi} B_{c}^{\frac{\alpha}{2}\left(\mu+\frac{1}{2}\right)}}\left(\frac{1}{2 \pi l}\right)^{2} \\
& \int_{L 1} \int_{L 2} \Gamma(\xi 1) *\left(\frac{2 \mu(h-H)}{\tilde{\gamma}_{i}^{\frac{\alpha}{2}}}\right)^{-\xi 1} \\
& \times \frac{\Gamma\left(\mu-\frac{1}{2}+\xi 2\right) \Gamma\left(\frac{1}{2}-\xi 2\right)}{\Gamma\left(\frac{1}{2}+\mu-\xi 2\right)}\left(\frac{4 \mu H}{\tilde{\gamma}_{i}^{\frac{\alpha}{2}}}\right)^{-\xi 2} \\
& \times \frac{\Gamma\left(\frac{\alpha}{2}\left(\mu+\frac{1}{2}-\xi 1-\xi 2\right)\right)}{\Gamma\left(\frac{\alpha}{2}\left(\mu+\frac{1}{2}-\xi 1-\xi 2\right)+1\right)} \\
& \times \Gamma\left(\frac{\alpha}{2}\left(\mu+\frac{1}{2}-\xi 1-\xi 2\right)+\frac{1}{2}\right) d \xi 1 d \xi_{2},
\end{aligned}
$$

where $A_{c}$ and $B_{c}$ are defined based on the modulation technique, where they can be found in table (I) in [2], and $\mathscr{A}_{i}=\frac{\alpha \mu^{\mu+\frac{1}{2}} h^{\mu}}{H^{\mu-\frac{1}{2}} \Gamma(\mu) \tilde{r}_{i}^{\frac{\alpha}{2}\left(\mu+\frac{1}{2}\right)}} \cdot$ In addition, equation (11) can be expressed in form of $H_{p 1, q 1: p 2, q 2 ; p 3, q 3}^{m 1, n 1 ; ., n]}$ for the bivariate $\mathrm{H}_{-}$ Fox function as in equation (8) according to its definition in [12, eq. 2.57].

\section{B. Average BER of U2 ( Cell Center User) over $\alpha-\eta-\mu$ Fading Channel:}

Now, we compute the average BER of the cell center user (U2) by submitting equation (2) and (10) into equation (3), and then follow the similar derivation steps from (5) to (11) in the previous subsection (A). Thus, the ABER of the cell center user becomes like:

$$
\begin{aligned}
\bar{P}_{U 2}(e) & =\frac{1}{2} \times \sum_{i i=1}^{5} \frac{A_{c} \mathscr{A}_{i i}}{\sqrt{\pi} B_{c}^{\frac{\alpha}{2}\left(\mu+\frac{1}{2}\right)}}\left(\frac{1}{2 \pi l}\right)^{2} \\
& \int_{L 1} \int_{L 2} \Gamma(\xi 1) *\left(\frac{2 \mu(h-H)}{\tilde{\gamma}_{i i}^{\frac{\alpha}{2}}}\right)^{-\xi 1} \\
& \times \frac{\Gamma\left(\mu-\frac{1}{2}+\xi 2\right) \Gamma\left(\frac{1}{2}-\xi 2\right)}{\Gamma\left(\frac{1}{2}+\mu-\xi 2\right)}\left(\frac{4 \mu H}{\tilde{\gamma}_{i i}^{\frac{\alpha}{2}}}\right)^{-\xi 2} \\
& \times \frac{\Gamma\left(\frac{\alpha}{2}\left(\mu+\frac{1}{2}-\xi 1-\xi 2\right)\right)}{\Gamma\left(\frac{\alpha}{2}\left(\mu+\frac{1}{2}-\xi 1-\xi 2\right)+1\right)} \\
& \times \Gamma\left(\frac{\alpha}{2}\left(\mu+\frac{1}{2}-\xi 1-\xi 2\right)+\frac{1}{2}\right) d \xi 1 d \xi_{2},
\end{aligned}
$$

where $i i \in\{1,2,3,4,5\}$, and $\tilde{\gamma}_{1}=\left(\sqrt{E_{s_{1}} / 2 \sigma_{2}^{2}}+\sqrt{E_{s_{2}} / 2 \sigma_{2}^{2}}\right)$ , $\tilde{\gamma}_{2}=\sqrt{\frac{E_{s_{2}}}{2 \sigma_{2}^{2}}}, \quad \tilde{\gamma}_{3}=2 \times\left(\sqrt{E_{s_{1}} / 2 \sigma_{2}^{2}}+\sqrt{E_{s_{2}} / 2 \sigma_{2}^{2}}\right) \quad, \quad \tilde{\gamma}_{4}=2 \times$ $\left(\sqrt{E_{s_{1}} / 2 \sigma_{2}^{2}}-\sqrt{E_{s_{2}} / 2 \sigma_{2}^{2}}\right), \tilde{\gamma}_{5}=\left(\sqrt{E_{s_{1}} / 2 \sigma_{2}^{2}}-\sqrt{E_{s_{2}} / 2 \sigma_{2}^{2}}\right)$, and $\mathscr{A}_{i i}=$ $\frac{\alpha \mu^{\mu+\frac{1}{2}} h^{\mu}}{H^{\mu-\frac{1}{2}} \Gamma(\mu) \tilde{\gamma}_{i i}^{\frac{\alpha}{2}\left(\mu+\frac{1}{2}\right)}}$. These different values of average signal to noise ratio $\left(\tilde{\gamma}_{i i}\right)$ are mainly based on the conditional probability distribution function (PDF) of users symbols in AWGN channel according to its superimposed symbol's energy over its constellation points as it can be shown as an example in [8]. However, the final form in bivariate H-Fox function can be seen in equation (9).

\section{Special Cases of $\alpha-\eta-\mu$ Fading Channel :}

According to [9], the $\alpha-\eta-\mu$ fading model contains special cases of other well known fading distributions such as Rayleigh fading that could be obtained from the final equation of ABER when $(\alpha=2, \eta=1, \mu=0.5)$. However, when these parameters are modified to be as follow: $(\alpha=2.5, \eta=1$, 
$\mu=0.5$ ), Weibull distribution will be produced. Moreover, Nakagami-m distribution will be matching if $(\alpha=2, \eta=1$, $\mu=1.25$ ). Finally, $\alpha-\mu$ distribution can be occurred when $(\alpha=2.5, \eta=1, \mu=1.25$ ). In figs. (4-5), the aforementioned fading distributions are shown clearly for both NOMA users.

\section{Numerical AND Simulation RESUlts:}

In this particular section, our intention is to study the effect of the fading components implemented the second format of $\alpha-\eta-\mu$ fading Channel. The Monte Carlo Simulations is plotted along with the analytical results to show the agreement between the numerical derivation and the simulation parts. In this scenario, we consider the unity of total transmission power with different fixed amount of power allocation coefficients for both user based upon the previous assumptions of channel conditions, which are considered as : $\left(\varepsilon_{1}=4 / 5, \varepsilon_{2}=1 / 5\right)$. As a consequence, fig. 1 shows the effect of $\alpha$ component with assigned range of values (from 0.5 to 0.75 ) for both users over $\alpha-\eta-\mu$ fading channel with format II while other parameters are considered to be constant as $(\eta=0.65, \mu=1.2)$. As a consequence, it can be seen from fig.1, that the increased rate in component $\alpha$ will significantly improve the BER of both users.

However, when we fix the following parameters $(\alpha=0.75$, $\mu=1.8$ ), and play around with $\eta$ component with different values (from 0.4 to 0.8 ), the system performance of both users will dramatically decreased whenever $\eta$ gets increased such as in fig.2 .

On the other hand, in fig.3, we set the values of $\alpha$ and $\eta$ parameters as $(\alpha=1.25, \eta=0.65)$ and allocate parameter $\mu$ to the following range (from 0.8 to 1.8 ). In this scenario, the overall BER of both users at high values of parameter $\mu$ will be interestingly enhanced. Therefor, it leads to the fact that the overall system performance is dramatically enhanced whenever the values of components $\alpha$ and $\mu$ are meant to be increased as long as $\eta$ value gets decreased.

From the opposed point of view, as it has been mentioned before that $\alpha-\eta-\mu$ fading channel encompasses other fading models as special cases that clearly has plotted in fig.4. It actually includes Rayleigh, Weibull, Nakagami-m, $\alpha-\eta-\mu$ and $\alpha-\mu$ fading channels for both users, and their assigned parameters are based on the previous subsection except the $\alpha-\eta-\mu$ fading which are as follow: $(\alpha=1.6, \eta=0.2, \mu=2)$. The result in fig.4 shows that $\alpha-\mu$ fading channels is the most better performance overall while the Rayleigh fading channel is considered as the worst case. Eventually, U2's BER performance is better than U1's BER performance since it experiences strength channel conditions than U1, even though it gets low amount of allocated power according to the NOMA protocol which leads in somehow to the fairness among different users.

\section{CONCLUSiON}

The performance analysis of DL-NOMA system operating over $\alpha-\eta-\mu$ Generalized Fading Channels of two users was characterized for the Average Bit Error Rate (ABER) metric.
Therefore, a closed form of each user in terms of Average Bit Error probability is analytically obtained and simulated. Moreover, the Monte Carlo Simulation has been applied to validate the numerical results of the fading parameters in different format of $\alpha-\eta-\mu$ fading model, and perfect matching is occurred in all cases. The simulation results show that the BER severity for both users is increased as $\eta$ grows while $\alpha$ and $\mu$ parameters are decreased. The incremental amount of multipath clusters beneficially contributes in terms of BER severity, and hence the bit error probability at low SNR is potentially decreased as $\alpha$ and $\mu$ are raised. Ultimately, the future work of this paper could be extended to investigating other system performance metrics and exemplifying the effect of these fading parameters on these potential metrics in different types of transmission system deployments.

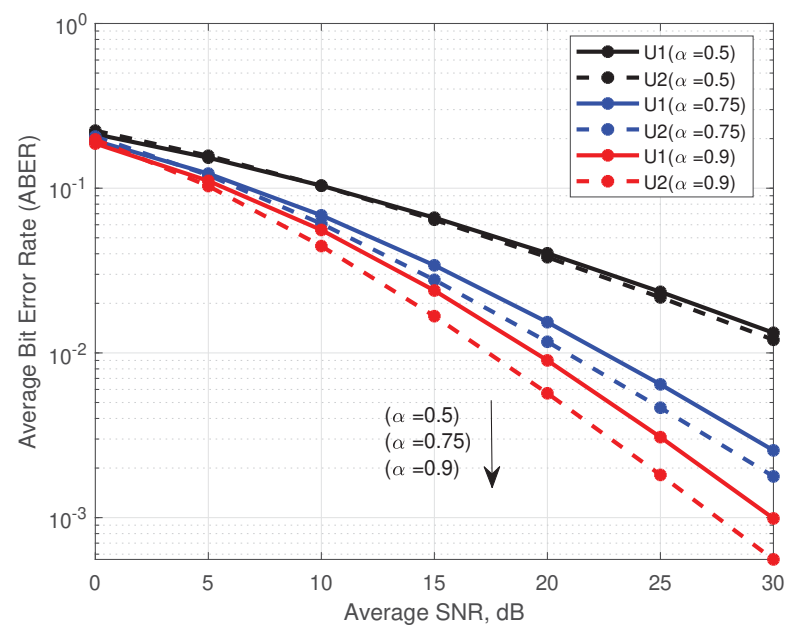

Fig. 1. ABER of Two NOMA users over $\alpha-\eta-\mu$ Fading Channel, Format II , with Fixed Values of $(\eta=0.65, \mu=1.2)$

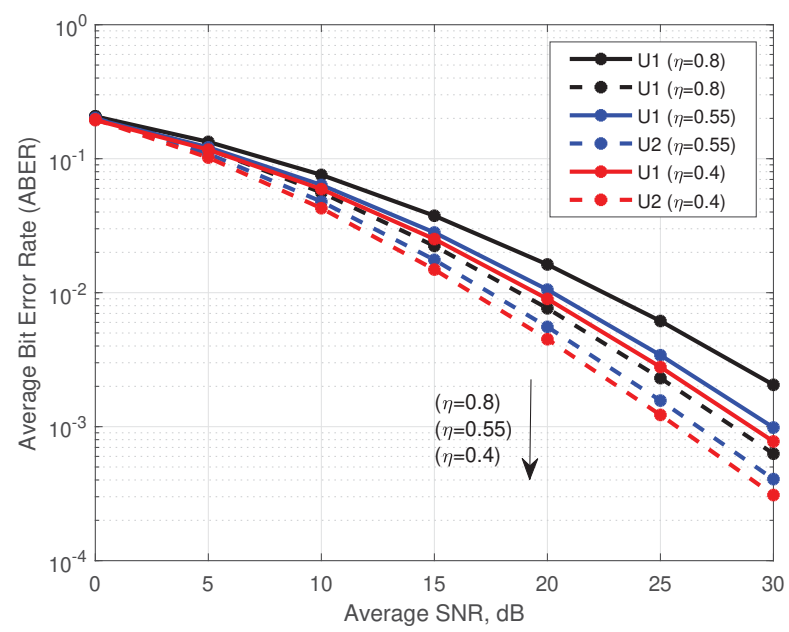

Fig. 2. ABER of Two NOMA users over $\alpha-\eta-\mu$ Fading Channel, Format II , with Fixed Values of $(\alpha=0.75, \mu=1.8)$ 


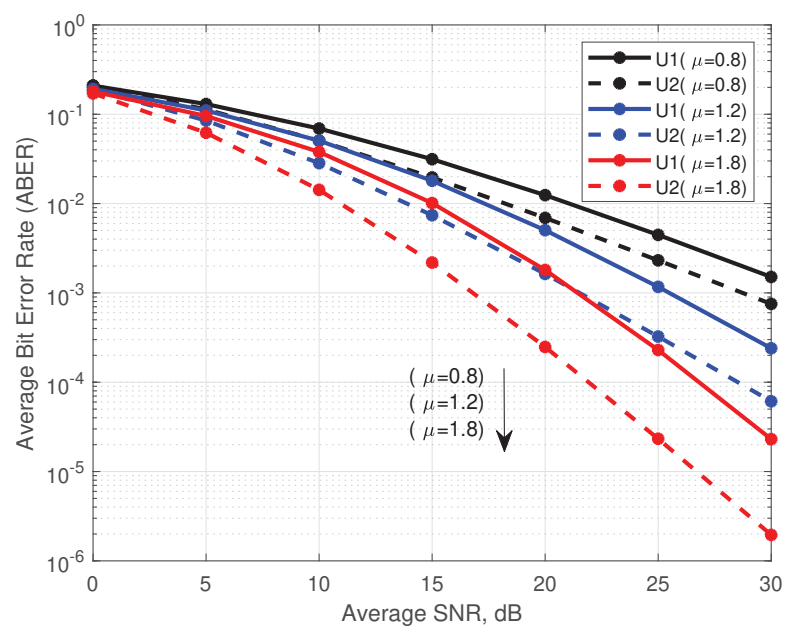

Fig. 3. ABER of Two NOMA users over $\alpha-\eta-\mu$ Fading Channel, Format II , with Fixed Values of $(\alpha=1.25, \eta=0.65)$

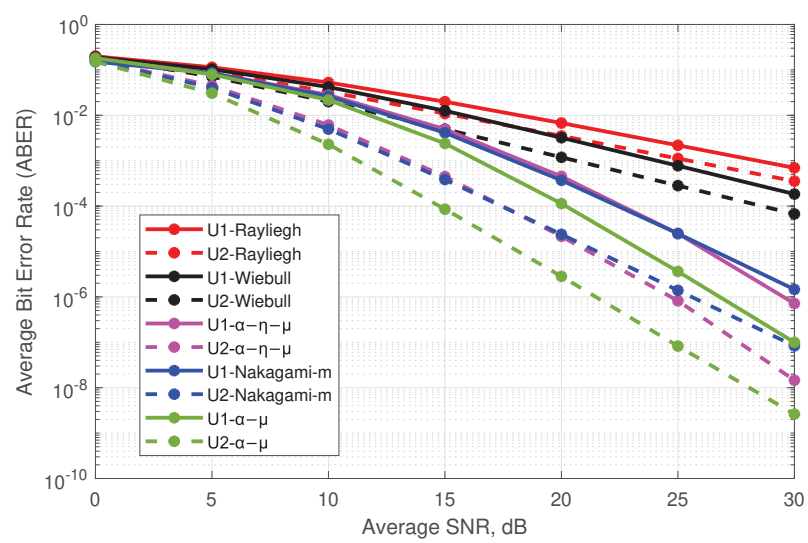

Fig. 4. ABER of Two NOMA users over different fading as special case of $\alpha-\eta-\mu$ Fading Channel, Format II

\section{REFERENCES}

[1] V. K. Upadhaya, P. S. Chauhan and S. K. Soni, "Effective Capacity Analysis over Generalized $\alpha-\eta-\mu$ fading channel," 2019 International Conference on Electrical, Electronics and Computer Engineering (UPCON), ALIGARH, India, 2019, pp. 1-3.

[2] O. S. Badarneh and M. S. Aloqlah, "Performance Analysis of Digital Communication Systems Over $\alpha-\eta-\mu$ Fading Channels," in IEEE Transactions on Vehicular Technology, vol. 65, no. 10, pp. 7972-7981, Oct. 2016.

[3] D. Morales-Jiménez and J. F. Paris, "Outage probability analysis for $\eta-\mu$ fading channels," IEEE Commun. Lett., vol. 14, no. 6, pp. 521-523, Jun. 2010.

[4] A. M. Magableh and M. M. Matalgah, "Moment generating function of the generalized $\alpha-\mu$ distribution with applications," IEEE Commun. Lett., vol. 13, no. 6, pp. 411-413, Jun. 2009.

[5] M. D. Yacoub, "The $\alpha-\mu$ Distribution: A Physical Fading Model for the Stacy Distribution," IEEE Trans. Veh. Technol., vol. 56, no. 1, pp. $27-$ 34, Jan. 2007

[6] M. D. Yacoub, "The $x-\mu$ Distribution and the $n-\mu$ Distribution," IEEE Antennas and Propagation Magazine, vol. 49, Feb. 2007.

[7] M. D. Yacoub and G. Fraidenraich, "The $\alpha-\eta-\mu$ and $\alpha-x-\mu$ Fading Distributions," IEEE Ninth International Symposium on Spread Spectrum Techniques and Applications, pp. 16 - 20, Aug. 2006.
[8] T. Assaf, A. Al-Dweik, M. E. Moursi and H. Zeineldin, "Exact BER Performance Analysis for Downlink NOMA Systems Over Nakagamim Fading Channels," in IEEE Access, vol. 7, pp. 134539-134555, 2019.

[9] Adriano Amaral de Souza, Rausley \& Ribeiro, Antônio \& Guimarães, Dayan. "On the Efficient Generation of $\alpha-x-\mu$ and $\alpha-\eta-\mu$ White Samples with Applications", in International Journal of Antennas and Propagation, 2015.

[10] A. P. Prudnikov, Y. A. Brychkov, and O. I. Marichev, Integrals and Series: More Special Functions, vol. 3. New York, NY, USA: Gordon \& Breach, 1990.

[11] M. K. Simon and M. Alouini, Digital Communication Over Fading Channels, 2nd ed. New York, NY, USA: Wiley, 2005.

[12] Mathai, A. M., Saxena, Ram Kishore, Haubold, Hans J., The H-Function, New York, NY. : Springer, 2015, pp. 60-62. 